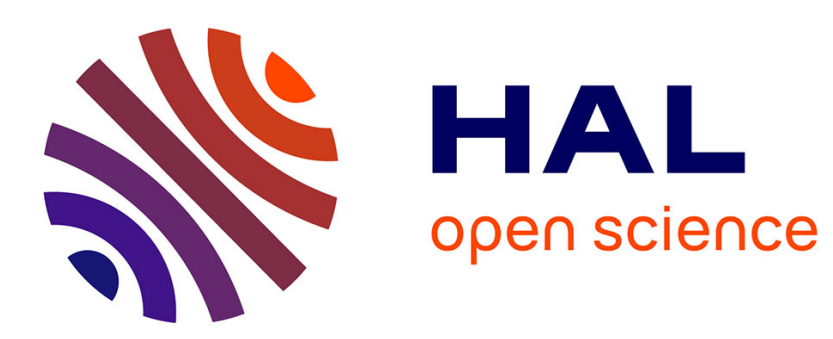

\title{
Help when needed, but no more: Efficient Read/Write Partial Snapshot
}

Damien Imbs, Michel Raynal

\section{To cite this version:}

Damien Imbs, Michel Raynal. Help when needed, but no more: Efficient Read/Write Partial Snapshot. [Research Report] PI 1907, 2008, pp.26. inria-00339292

\section{HAL Id: inria-00339292 \\ https://hal.inria.fr/inria-00339292}

Submitted on 17 Nov 2008

HAL is a multi-disciplinary open access archive for the deposit and dissemination of scientific research documents, whether they are published or not. The documents may come from teaching and research institutions in France or abroad, or from public or private research centers.
L'archive ouverte pluridisciplinaire HAL, est destinée au dépôt et à la diffusion de documents scientifiques de niveau recherche, publiés ou non, émanant des établissements d'enseignement et de recherche français ou étrangers, des laboratoires publics ou privés. 


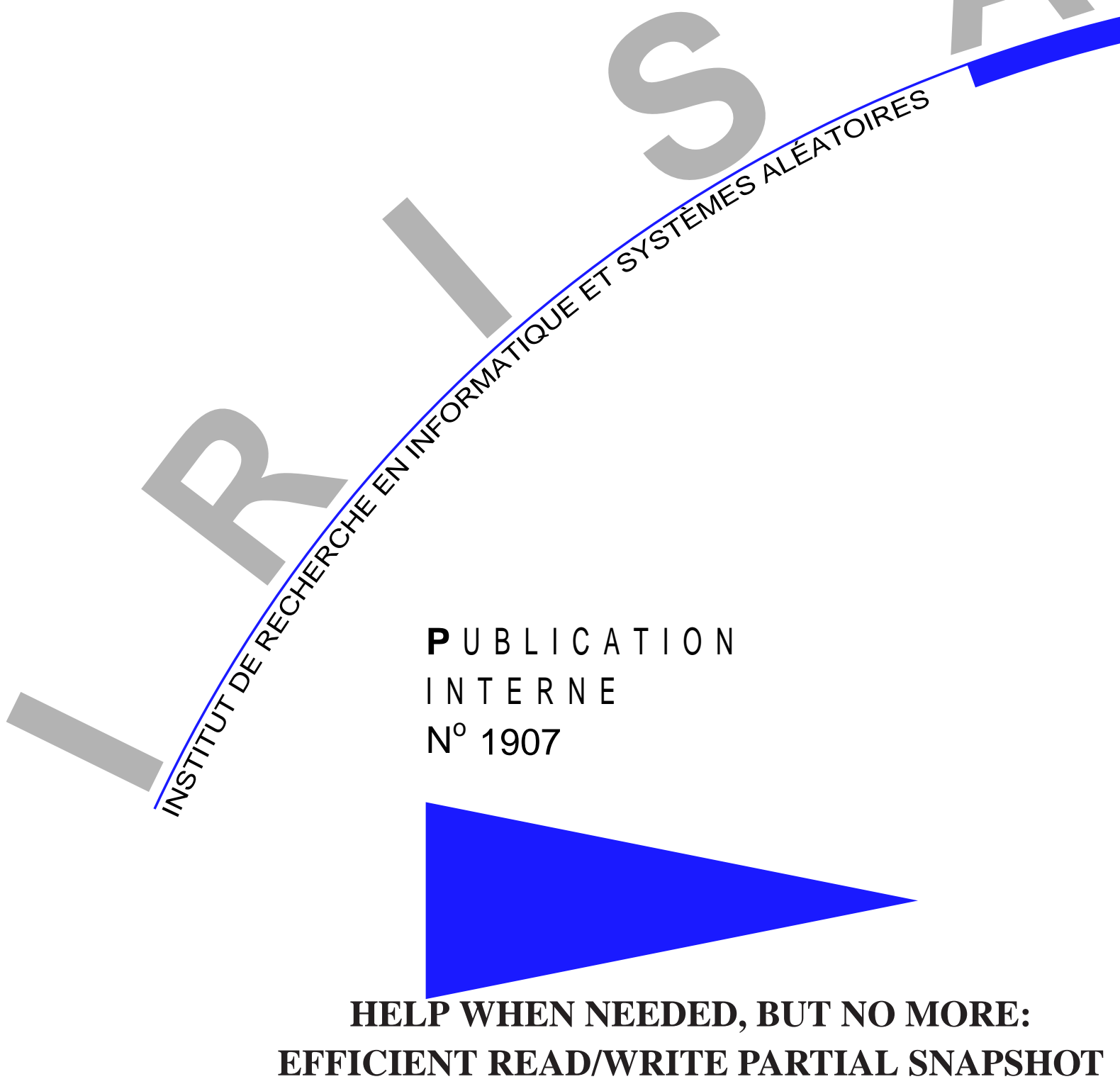

DAMIEN IMBS MICHEL RAYNAL 



\title{
Help when needed, but no more: Efficient Read/Write Partial Snapshot
}

\author{
Damien Imbs $^{*} \quad$ Michel Raynal ${ }^{* *}$ \\ Systèmes communicants \\ Projet ASAP \\ Publication interne $\mathrm{n}^{\circ} 1907$ - Novembre 2008 - 24 pages
}

\begin{abstract}
An atomic snapshot object is an object that can be concurrently accessed by asynchronous processes prone to crash. It is made of $m$ components (base atomic registers) and is defined by two operations: an update operation that allows a process to atomically assign a new value to a component and a snapshot operation that atomically reads and returns the values of all the components. To cope with the net effect of concurrency, asynchrony and failures, the algorithm implementing the update operation has to help concurrent snapshot operations in order they can always terminate.

This paper is on partial snapshot objects. Such an object provides a snapshot operation that takes a (dynamically defined) subset of the components as input parameter, and atomically reads and returns the values of this subset of components. The paper has two contributions. The first is the introduction of two properties for partial snapshot object algorithms, called help-locality and uptodateness. Help-locality requires that an update operation helps only the concurrent partial snapshot operations that read the component it writes. When an update of a component $r$ helps a partial snapshot, uptodateness requires that the update provides the partial snapshot with a value of the component $r$ that is at least as recent as the value it writes into that component. (No snapshot algorithm proposed so far satisfies these properties.) The second contribution consists of an update and a partial snapshot algorithms that are wait-free, linearizable and satisfy the previous efficiency properties. Interestingly, the principle that underlies the proposed algorithms is different from the one used so far, namely, it is based on the "write first, and help later" strategy. An improvement of the previous algorithms is also presented. Based on LL/SC atomic registers (instead of read/write registers) this improvement decreases the number of base registers from $O\left(n^{2}\right)$ to $O(n)$. This shows an interesting tradeoff relating the synchronization power of the base operations and the number of base atomic registers when using the "write first, and help later" strategy.
\end{abstract}

Key-words: Adaptive algorithm, Asynchronous shared memory system, Asynchrony, Atomicity, Atomic snapshot, Efficiency, Concurrency, Linearizability, LL/SC atomic registers, Locality, Partial snapshot, Process crash, Read/Write atomic register, Wait-free algorithm.

(Résumé : tsvp)

* IRISA, Université de Rennes 1, Campus de Beaulieu, 35042 Rennes Cedex, France damien.imbs @irisa.fr

** IRISA, Université de Rennes 1, Campus de Beaulieu, 35042 Rennes Cedex, France, raynal@irisa.fr

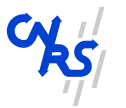

Centre National de la Recherche Scientifique (UMR 6074) Université de Rennes 1 - Insa de Rennes
Institut National de Recherche en Informatique et en Automatique - unité de recherche de Rennes 


\section{N'aider que si c'est nćessaire : Capture efficace d'instantanés partiels}

Résumé : Ce rapport présente un algorithme efficace pour la lecture atomique d'un ensemble de variables partagées par des processus concurrents sujets à la défaillance par crash.

Mots clés : Atomicité, Contrôle de la concurrence, Crash de processus, Mémoire partagée asynchrone, Capture d'instantané, Linéarizabilité, Registre atomique, Opérations LL/SC, Localité, Synchronisation sans attente. 


\section{Introduction}

\subsection{Context of the study: snapshot objects}

Shared memory snapshot objects Snapshot objects have been introduced in [1]. Considering a shared memory system made up of base atomic read/write registers, that can be concurrently accessed by asynchronous processes prone to crash, a snapshot object is an object that (1) consists of $m$ components (each component being a base atomic register that can contain an arbitrary value), and (2) provides the processes with two operations, denoted update() and snapshot(). The update() operation allows the invoking process to atomically store a new value in an individual component. Differently, the snapshot () operation returns the values of all the components as if they had been read simultaneously.

From an execution point of view, a snapshot object has to satisfy the safety property called linearizability: the update and snapshot operations have to appear as if they had been executed one after the other, each being instantaneously executed at some point of the time line comprised between its start event and its end event [19]. From a liveness point of view, each update or snapshot operation has to terminate if the invoking process does not crash. This liveness property is called wait-freedom [16]. It means that an operation issued by a correct process has to terminate whatever the behavior of the other processes (the fact that some processes crash or are very slow cannot prevent an operation from terminating, as long as the issuing process does not crash). Wait-freedom is starvation-freedom despite asynchrony and process failures. In order to implement the wait-freedom property, a process that issues an update() operation can be required to help terminate the processes that have concurrently issued a snapshot () operation (preventing them from looping forever). This helping mechanism is required to ensure that all the snapshot() operations (issued by processes that do not crash) do always terminate [1].

The snapshot abstraction The snapshot object has proved to be a very useful abstraction for solving many other problems in asynchronous shared memory systems prone to process crashes, such as approximate agreement, randomized consensus, concurrent data structures, etc. A snapshot object hides the "implementation details" that are difficult to cope with in presence of the net effect of concurrency, asynchrony and failures. It is important to notice that, from a computational point of view, a snapshot object is not more powerful than the base atomic read/write objects it is built from. It only provides a higher abstraction level.

Shared memory snapshot vs message-passing snapshot The values returned by a snapshot() operation is a value of the part of the shared memory that is encapsulated in the corresponding snapshot object. It follows from the linearizability property satisfied by a snapshot object that there is a time instant at which the values returned by a snapshot() operation were simultaneously present in the shared memory, this time instant belonging to the time interval associated with that snapshot() operation.

The previous observation is in contrast with the notion of distributed snapshot used to capture consistent global states in asynchronous message-passing systems [11] where two distributed snapshots obtained by two processes can be consistent but incomparable in the sense that they cannot be linearized. The set of all the distributed snapshots that can be obtained from a message-passing distributed execution has only a lattice structure (basically, they can be partially ordered but not totally ordered). In that sense, the abstraction level provided by a shared memory snapshot object is a higher abstraction level than the one offered by messagepassing distributed snapshots. They hide more asynchrony.

Types of snapshot objects Two types of snapshot objects have been investigated: single-writer and multiwriter snapshot objects. A single-writer snapshot object has one component per process, and the component associated with a process can be written only by that process. The number of components $(m)$ is then 
the same as the number of processes $(n)$. The base registers from which a single-writer snapshot object is built are then single-writer/multi-reader atomic registers. Wait-free algorithms implementing single-writer snapshot objects for $n$ processes are described in [1]. Their costs is $O\left(n^{2}\right)$ (when counting the number of shared memory accesses). An algorithm whose cost is $O(n \log (n))$ is described in [9]. An implementation suited to systems with a possibly infinite number of processes (but where finitely many processes can take steps in each finite time interval) is described in [3]. An implementation that is adaptive to total contention (i.e., adaptive to the actual number $k \in[1 . . n]$ of processes that access the snapshot object during an entire execution [6]) is described in [7]. Its cost is $O(k \log (k))$.

A multi-writer snapshot object is a snapshot object of which each component can be written by any process. So, the base read/write registers on which its implementation relies are multi-writer/multi-reader atomic registers. Wait-free algorithms implementing multi-writer snapshot objects made up of $m$ base components are described in [4, 21, 22]. The algorithm described in [21] has a linear cost $O(n)$. A short survey of algorithms that implement single-writer and multi-writer snapshot objects is presented in [12].

The notion of partial snapshot Usually, when a process invokes the snapshot () operation, it is not interested in obtaining the values of all the components, but in the values of a given subset of the components. A partial snapshot operation (denoted p_snapshot()) is a generalization of the base snapshot () operation. It takes a sequence $R=<r_{1}, \cdots, r_{x}>$ of component indices as input parameter, and returns a sequence $\left\langle v_{1}, \cdots, v_{x}>\right.$ of values such that the value $v_{\ell}$ is the value of the component whose index is $r_{\ell}$ (an invocation of p_snapshot () that considers all the components is actually a snapshot() invocation). As before, the invocations of p_snapshot() and update() have to be linearizable, and their implementation has to be wait-free. The notion of partial snapshot object has first been introduced and investigated in [8].

\subsection{Content of the paper and related work}

Related work This paper is on the efficient wait-free implementation of multi-writer/multi-reader partial snapshot objects in the base read/write shared memory model augmented with an underlying active set object [2]. Such an object offers three operations: Join(), Leave() and GetSet(). Basically, Join() adds the invoking process to the active set, while Leave() suppresses it from this set; GetSet() returns the current value of the active set. (There are efficient adaptive read/write implementations of an active set, i.e., implementations whose number of read/write shared memory accesses depends only on the number of processes that invoke Join() and Leave() [2]. So, the base model used in this paper is the read/write atomic register model.

An algorithm based on read/write atomic registers and an active set object, that implements a partial snapshot object is described in [8] (as far as we know, it is the only such algorithm proposed so far) ${ }^{1}$. That algorithm extends the basic full snapshot algorithm described in [1]. It is based on the following principle. Each invocation of p_snapshot $(R)$ first makes public the list $R=<r_{1}, \cdots, r_{x}>$ of indices of the components it wants to read. Then, it sequentially invokes Join(), an internal embedded_snapshot $(R)$ operation, and finally Leave(). The update operation works as follows. When a process $p_{i}$ invokes update $(r, v, i)$ (where $v$ is the value it wants to assign to the component whose index is $r$ ), it first invokes GetSet() to have a view of all the processes that are concurrently executing a p_snapshot() operation. To guarantee the wait-freedom property (any process that does not crash has to terminate its operations), $p_{i}$ helps terminate all the concurrent p_snapshot() operations. To that end, it executes an embedded_snapshot() operation whose input includes all the components read by the processes in the active set (whose value has been obtained by

\footnotetext{
${ }^{1}$ That paper presents also another algorithm implementing a partial snapshot object, that is based on read/write atomic registers, and more sophisticated registers that support Fetch\&Add and Compare\&Swap atomic operations. These more sophisticated registers are mainly used to obtain an efficient implementation of the underlying active set object. Here we consider the pure base $\mathrm{read} / \mathrm{write}$ atomic register model.
} 
the GetSet() invocation). In that way, if $p_{i}$ does not crash, a concurrent p_snapshot() operation can retrieve the values it is interested in from the values returned by the embedded_snapshot () issued by $p_{i}$.

Features of the proposed algorithm The update and partial snapshot algorithms proposed here have several noteworthy features that make them different from all the previous full/partial snapshot algorithms (as far as we know). These features are the "write first, help later" strategy, and the cheap way helping is realized. They result from the additional help-locality and uptodateness properties the update and snapshot algorithms are required to satisfy.

Uptodateness The aim of the uptodateness property is to oblige an update operation that helps a snapshot operation to provide that snapshot with values as recent as possible. More precisely, let up $=$ update $(r, v, i)$ be an update invoked by the process $p_{i}$ to write the value $v$ in the component $r$ of the partial snapshot object, and $\mathrm{psp}=$ p_snapshot $(R)$ be a concurrent snapshot invocation such that $r \in R$. Moreover, let us suppose that psp is helped by up, i.e., the values returned by psp have been provided by up. Uptodateness requires that the value returned for the component $r$ be $v$ or a more recent value (as each component is an atomic register, the notion of "more recent" is well defined) ${ }^{2}$.

To obtain that property, the update algorithm proposed in the paper uses the "first write, help later" strategy (differently, the previous algorithms are based on the "help first, then write" strategy). As it allows providing a snapshot with more uptodate values, the uptodateness property shows that the unusual "write first, help later" strategy is really interesting and should maybe deserve more investigation.

Help-locality This property aims at obtaining more efficient update operations. To that end, it reduces the help provided to the partial snapshot operations by the update operations. More explicitly, let update $(r,-,-)$ be an update operation that is concurrent with a partial snapshot operation $p_{\text {_snapshot }}(R)$. The help-locality property demands the update not to help the partial snapshot if they do not conflict, i.e., if $r \notin R$. This means that, when update $(r,-,-)$ is concurrent with p_snapshot $\left(R_{1}\right), \ldots$, p_snapshot $\left(R_{z}\right)$, it has to help only the ones such that $r \in R_{\ell}(1 \leq \ell \leq z)$. This favors disjoint access parallelism.

As for uptodateness, the help-locality property is not ensured by the algorithms proposed so far to implement the update operation. The snapshot algorithm presented in [1] is very conservative: each update operation is required to compute one helping full snapshot value even when there is no concurrent snapshot. The partial snapshot algorithm described in [8] is a little bit less conservative: an update $(r,-,-)$ operation concurrent with no snapshot operation is not required to help, but an update $(r,-,-)$ operation concurrent with one or more p_snapshot $\left(R_{\ell}\right)$ operations $(1 \leq \ell \leq z)$ has to help each of them, whatever the sets $R_{\ell}$, i.e., even the p_snapshot $\left(R_{\ell}\right)$ operations such that $r \notin R_{\ell}$.

An additional asynchrony feature An additional feature of the proposed update algorithm lies in its asynchrony and in the size of the helping snapshot values it computes. Previous (partial or full) snapshot implementations use one base atomic register $R E G[r]$ per component $r$. These registers have to be large. They are made up of several fields, including a field for the last value written, a field storing a snapshot value used to help snapshot operations, and a few other fields containing control data. A snapshot value is made up of one value per component in the case of a full snapshot object, and one value for a subset of the components in the case of a partial snapshot object. Then, each update $(r, v,-)$ operation atomically writes

\footnotetext{
${ }^{2}$ It is important to notice that neither the full snapshot algorithms proposed so far (e.g., [1]), nor the partial snapshot algorithm presented in [8], satisfies the uptodateness property. They all provide the snapshot with a component $r$ value that is strictly older than $v$.
} 
into $R E G[r]$ both the new value $v$ and a snapshot value. This means that the implementation of this atomic write can be time expensive.

Differently, thanks to the "write first, help later (and individually)" strategy, the proposed update $(r, v,-)$ algorithm separates the write of the value $v$ into $R E G[r]$ and the individual writes of helping snapshot values, one for each concurrent p_snapshot $\left(R_{\ell}\right)$ operation such that $r \in R_{\ell}$. The fact that an update $(r, v,-)$ operation first writes $v$, and helps, only later and individually, each concurrent partial snapshot that reads the component $r$, (1) allows those to obtain a value for the component $r$ that is at least as recent as $v$, and (2) allows the use of several independent helping atomic registers that are written individually (thereby allowing more efficient atomic write operations). Moreover, the size of these atomic "array-like" registers can be smaller than $m^{3}$.

Motivation As the work described in [8], our aim is to better understand synchronization in presence of failures. From a more practical point of view, a p_snapshot $(R)$ operation can be seen as the reading part of a transaction that needs to obtain mutually consistent and uptodate values from the base objects specified in $R$. Such a study can help better understand the underlying foundations of software transactional memories $[5,13,14,18,17,20,25]$.

Roadmap The paper is composed of 7 sections. Section 2 presents the base asynchronous read/write shared memory prone to process crashes, equipped with an active set object. Section 3 defines the atomic partial snapshot object and the help-locality and uptodateness properties. Then, Section 4 presents algorithms implementing the update and partial snapshot operations. These algorithms satisfy the previous properties. They are proved correct in Section 5. Section 6 discusses the proposed algorithms and presents a version of them based on LL/SC atomic registers (instead of read/write atomic registers). This improvement, that satisfies the help-locality and uptodateness properties, is more efficient than the base algorithm from a memory size point of view, namely it requires $O(n) \mathrm{LL} / \mathrm{SC}$ atomic registers instead of $O\left(n^{2}\right) \mathrm{read} / \mathrm{write}$ atomic registers. Finally, Section 7 provides a few concluding remarks.

\section{Underlying shared memory model}

\subsection{Asynchronous shared memory model}

The system is made up of $n$ processes $p_{1}, \ldots, p_{n}$. The identity of $p_{i}$ is $i$. These processes communicate through multi-writer/multi-reader atomic registers. Atomic means that each read or write operation on a register appears as if it has been executed sequentially at some point of the time line comprised between its start and end event. The registers are assumed to be reliable (this assumption is without loss of generality -from a computability point of view- as it is possible to build atomic reliable registers on top of crash prone atomic registers $[10,15,17,23])$.

There is no assumption on the speed of processes: they are asynchronous. Moreover, up to $(n-1)$ processes may crash. Before it crashes (if it ever crashes), a process executes correctly its algorithm. A crash is a premature halt: after it has crashed, a process executes no more step. Given a run, a process that does not crash is correct in that run, otherwise it is faulty in that run.

\footnotetext{
${ }^{3}$ If each partial snapshot by a process $p_{i}$ is on at most $k$ components, the atomic "array-like" registers used to help $p_{i}$ need to have only $k$ entries. If $k<<m$, the writes into such atomic $k$-size registers can generate less contention that writes into atomic $m$-size registers.
} 


\subsection{Active set object}

We assume that the processes can access an active set object. Such an object, first proposed in [2], can be used to solve adaptive synchronization problems (e.g., [26]). As already indicated, its aim is to allow the processes to have a view of which of them are concurrently executing operations. To that end, an active set object provides the processes with three operations, Join(), Leave() and GetSet() (informally described in the Introduction). These operations are not required to be atomic. (So, the definition of an operation cannot assume that the concurrent executions of other operations are both instantaneous and one at a time, they have to explicitly take into account the fact that their execution spans a finite period of time.)

Let $S$ be an active set object. Initially, the predicate $i \notin S$ is true for any process $p_{i}$ ( $S$ is empty). A process $p_{i}$ executes the following sequence (expressed using the regular language notation) of operation invocations (from which the object identifier $S$ is omitted):

$$
\left[\operatorname{GetSet}()^{*}(\operatorname{Join}() \text { Leave }())^{*}\right]^{*} \text {. }
$$

- Let us consider two consecutive operations Join() and Leave() (if any) issued by a process $p_{i}$. (This means that (1) Leave() follows immediately Join(), or (2) Join() follows Leave() and only GetSet ()* can occur between them.)

- From the end event of Join() until the start event of Leave(), the predicate $i \in S$ is true.

- From the end event of Leave() until the start event of Join(), the predicate $i \in S$ is false.

- During the execution of Join() or Leave(), the predicate can be true or false.

- A GetSet() invocation returns a set of process ids including:

- Each $j$ such that the predicate $j \in S$ was continuously true during the execution of GetSet().

- No $j$ such that the predicate $j \in S$ was continuously false during the execution of GetSet().

- Possibly some $j$ s such that the predicate $j \in S$ was not continuously true during GetSet().

As an example let us consider the GetSet() invocation depicted in Figure 1 (the length of a box indicates the time duration of the corresponding operation). That GetSet() invocation returns a set that (1) does contain $k$ and does not contain $j$, and (2) can possibly contain $\ell$ or $m$.

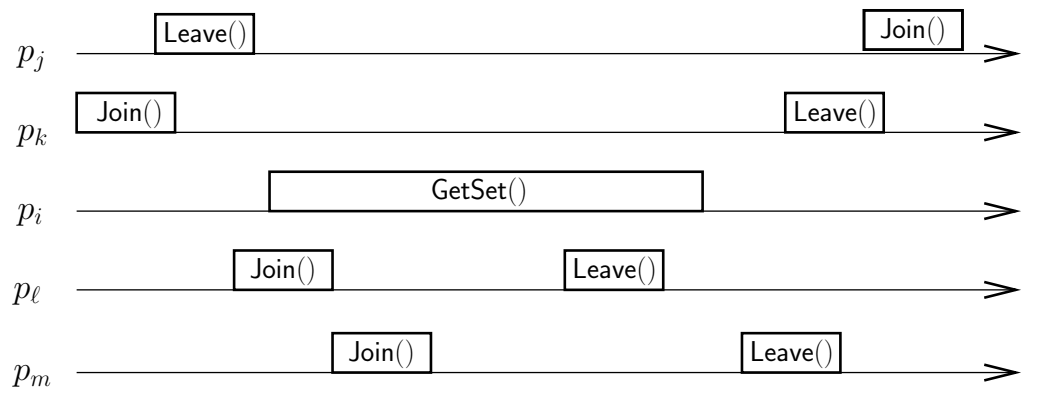

Figure 1: An example of active set object

An adaptive wait-free implementation of an active set object is described in [2]. Adaptive means that the cost of each operation (measured by the number of shared memory accesses) depends on the number of processes that have invoked Join() and have not yet terminated the corresponding Leave(). As already indicated, wait-free means that any operation invocation issued by a correct process always terminates [16]. PI ${ }^{\circ} 1907$ 


\section{Definitions}

\subsection{Partial snapshot object}

As already said in the Introduction, a multi-writer/multi-reader partial snapshot object is made up of $m$ components (each being a multi-writer/multi-reader atomic register) that provides the processes with two operations update () and snapshot () such that:

- update $(r, v, i)$ is invoked by $p_{i}$ to write the value $v$ in the component $r(1 \leq r \leq m)$ of the snapshot object. That operation returns the control value $o k$.

- p_snapshot $(R)$, where $R$ is a sequence $\left\langle r_{1}, \cdots, r_{x}>\right.$ of component indexes, allows a process to obtain the value of each component in $R$. It returns a corresponding sequence of values $\left\langle v_{1}, \cdots, v_{x}\right\rangle$.

A partial snapshot object is defined by the following properties.

- Termination. Every invocation of update() or p_snapshot() issued by a correct process terminates.

- Consistency. The operations issued by the processes (except possibly the last operation issued by a faulty process ${ }^{4}$ ) appear as if they have been executed one after the other, each one being executed at some point of the time line between its start event and its end event.

The termination property is wait-freedom [16] (starvation-freedom despite concurrency and process crashes). The consistency property is linearizability [19] (here, it means that a p_snapshot() operation always returns component values that were simultaneously present in the shared memory, and are uptodate).

\subsection{Additional properties related to the implementation}

These properties, that have been informally presented in the Introduction, do not concern the definition of the partial snapshot problem, but the way it is solved by the algorithms that implement its operations.

Definition 1 The algorithms implementing the update and partial snapshot operations satisfy the helplocality property if, for any pair $(r, R)$ such that $r \notin R$, an update $(r,-,-)$ invocation never helps a p_snapshot $(R)$ operation.

This property, related to efficiency, follows from the observation that an update $(r,-,-)$ operation and a p_snapshot $(R)$ operation that are concurrent and such that $r \notin R$, are actually independent operations. (This is similar to a read on a register $X$ and a write on a register $Y \neq X$ that are concurrent.) Intuitively, help-locality requires that the implementation does only what is necessary and sufficient.

Let p_snapshot $\left(\left\langle r_{1}, \ldots, r_{n}\right\rangle\right)$ be a snapshot operation that returns a snapshot value $\left\langle v_{1}, \ldots, v_{n}\right\rangle$ that has been computed by an update operation, say update $(r, v,-)$. Hence, $r \in\left\{r_{1}, \ldots, r_{n}\right\}$ and the update helps the snapshot.

Definition 2 The algorithms implementing the update and partial snapshot operations satisfy the uptodateness property if, for a snapshot operation $\mathrm{p} \_$snapshot $(R)$ that is helped by an update $(r, v,-)$ operation, the value returned for the component $r$ is at least as recent as $v$.

The aim of this property is to provide the partial snapshot operations with values "as fresh as possible". As noticed in the Introduction, (to our knowledge) no pair of update/snapshot algorithms proposed so far satisfies help-locality or uptodateness.

\footnotetext{
${ }^{4}$ If such an operation does not appear in the sequence, it is as if it has not been invoked.
} 


\section{An efficient partial snapshot construction}

This section presents a construction (Figures 2 and 3) of a partial snapshot object that satisfies the helplocality and uptodateness properties previously defined.

\subsection{The underlying shared objects}

The algorithms implementing the p_snapshot () and update() operations use the following shared variables.

- An array, denoted $R E G[1 . . m]$, of multi-writer/multi-reader atomic registers. The register $R E G[r]$ is associated with the component $r$ of the snapshot object. It is composed of three fields $\langle$ value,pid, sn $\rangle$, whose meaning is the following. REG[r].value contains the current value of the component $r$; $R E G[r] . p i d$ and $R E G[r] . s n$ are control data associated with that value. $R E G[r] . p i d$ contains the id of the process that issued the corresponding update() operation, while $R E G[r]$.sn contains its sequence number among all the update() operations issued by that process.

- An array, denoted $A N N O U N C E[1 . . n]$, of single-writer/multi-reader atomic registers. The register $A N N O U N C E[i]$ can be written only by $p_{i}$. This occurs when $p_{i}$ invokes p_snapshot $(R)$ : it then stores $R$ in $A N N O U N C E[i]$ (the indexes $r_{1}, \cdots, r_{x}$ of the components it wants to read). In that way, if a process $p_{j}$ has to help $p_{i}$ to terminate its p_snapshot() operation, it only has to read ANNOUNCE[i] to know the components $p_{i}$ is interested in.

- An array, denoted HELPSNAP[1..n, 1..n], of single-writer/multi-reader atomic registers. The register HELPSNAP $[i, j]$ can be written only by $p_{i}$. When, while executing an update() operation, $p_{i}$ is required to help $p_{j}$ terminate its current p_snapshot $\left(\left\langle r_{1}, \cdots, r_{x}\right\rangle\right)$ operation, it does so by depositing in HELPSNAP $[i, j]$ a sequence of values $\left\langle v_{1}, \cdots, v_{x}\right\rangle$ that can be used by $p_{i}$ as the result of its p_snapshot $\left(<r_{1}, \cdots, r_{x}>\right)$ operation.

The shared variables are denoted with upper case letters. Differently, the local variables are denoted with lower case letters (those are introduced in the algorithm description).

\subsection{The p_snapshot () operation}

The algorithm that implements this operation is described in Figure 2. Similarly to [8], it borrows its underlying principle from [1]. More precisely, it first uses a "sequential double scan" to try to terminate by itself. If it cannot terminate by itself, it looks for a process that could help it terminate (namely, a process that has issued two updates on a component it wants to read).

Startup When it invokes p_snapshot $(R)$, a process $p_{i}$ first announces the components it wants to read (line 01) and invokes Join() (line 02). This is in order to allow the processes that concurrently update a component of $R$ to help it.

Sequential double scan Then, the process $p_{i}$ enters a loop (line 04-18). During each execution of the loop body, it uses a pair of scans of the registers $R E G[r]$ for the components it is interested in, namely $\left\{r_{1}, \ldots, r_{x}\right\}$. It is important to notice that these are sequential [1]: the second scan always starts after the previous one has terminated. The values obtained by the first scan are kept in the array aa (line 03 for the first loop, and then line 05 followed by line 17), while the values obtained by the second scan are kept in the array $b b$ (line 05). 


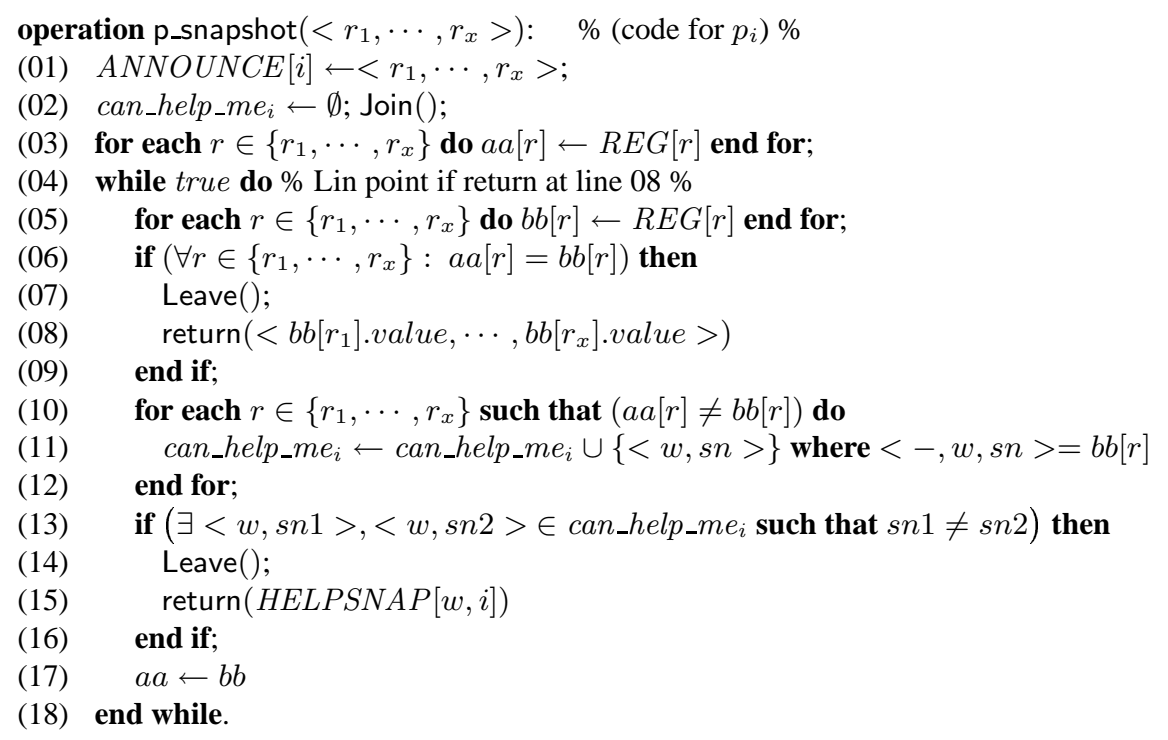

Figure 2: An algorithm for the p_snapshot() operation

Try first to terminate without help If, for each $r \in\left\{r_{1}, \ldots, r_{x}\right\}$, it observes no change in $R E G[r]$ (test of line 06), $p_{i}$ can conclude that at any point of the time line between the end of the first scan and the beginning of the second one, no $R E G[r], r \in\left\{r_{1}, \ldots, r_{x}\right\}$, has been modified. This is called a successful double scan. Hence, the values read in $b b$ were simultaneously present in the snapshot object: they can be returned as the result of the p_snapshot $\left(\left\langle r_{1}, \cdots, r_{x}\right\rangle\right)$ invocation (line 08). In that case, before terminating, $p_{i}$ invokes Leave() to announce that it does no longer need help (line 07).

Otherwise, try to benefit from the helping mechanism While until that point, the statements previously described are the same as the ones used in $[1,8]$, the statements that follow are different. This difference is mainly due to the "write first, then help" strategy, and its impact on the way it is exploited by the algorithm.

If the test of line 06 is not satisfied, $p_{i}$ uses the helping mechanism that (from its side) works as follows. As the test is false, there is at least one component $r \in\left\{r_{1}, \cdots, r_{x}\right\}$ that has been updated between the two scans. For each such component $r, p_{i}$ considers the identity of the last write, namely the pair $\langle w, s n\rangle$ extracted from $b b[r]=<-, w, s n>$ (the last writer of $R E G[r]$ is $p_{w}$ and $s n$ is the increasing sequence number it has associated with the corresponding update); $p_{i}$ adds this pair to a local set can_help_me $i$ where it stores the processes that could help it (lines 10-12).

Then, $p_{i}$ checks if it can terminate thanks to the helping mechanism (lines 13-16). The helping termination predicate is as follows: " $p_{i}$ has observed that there is a process $p_{w}$ that has issued two different updates (on any pair of components)". From an operational point of view, this is captured by the fact that $p_{w}$ appears twice in can_help_me $i$ (line 13). As we will see in the proof, the fact that this predicate is true means that $p_{w}$ has determined a set of values $\left\langle v_{1}, \cdots, v_{x}>(\right.$ kept in $\operatorname{HELPSNAP}[w, i])$ that $p_{i}$ can use as the result of its p_snapshot $\left.\left(<r_{1}, \cdots, r_{x}\right\rangle\right)$ operation. In that case, $p_{i}$ invokes Leave () to indicate it does no longer need help and returns the content of HELPSNAP $[w, i]$ (lines 14-15).

Finally, if the helping predicate is false, $p_{i}$ cannot terminate and consequently enters again the loop body (after having shifted the array $b b$ in the array $a a$, line 17). 


\subsection{The update() operation}

The algorithm for the update() operation is described in Figure 3. The invoking process $p_{i}$ first writes the new value (line 01 , where $n b w_{i}$ is a local sequence number generator), and then (lines 02-30) asynchronously helps the other processes. As indicated in the Introduction, the principles that underlie this mechanism differ from the ones used in previous snapshot/update algorithms. Let update $(r, v, i)$ be an update invocation. The helping mechanism works as follows.

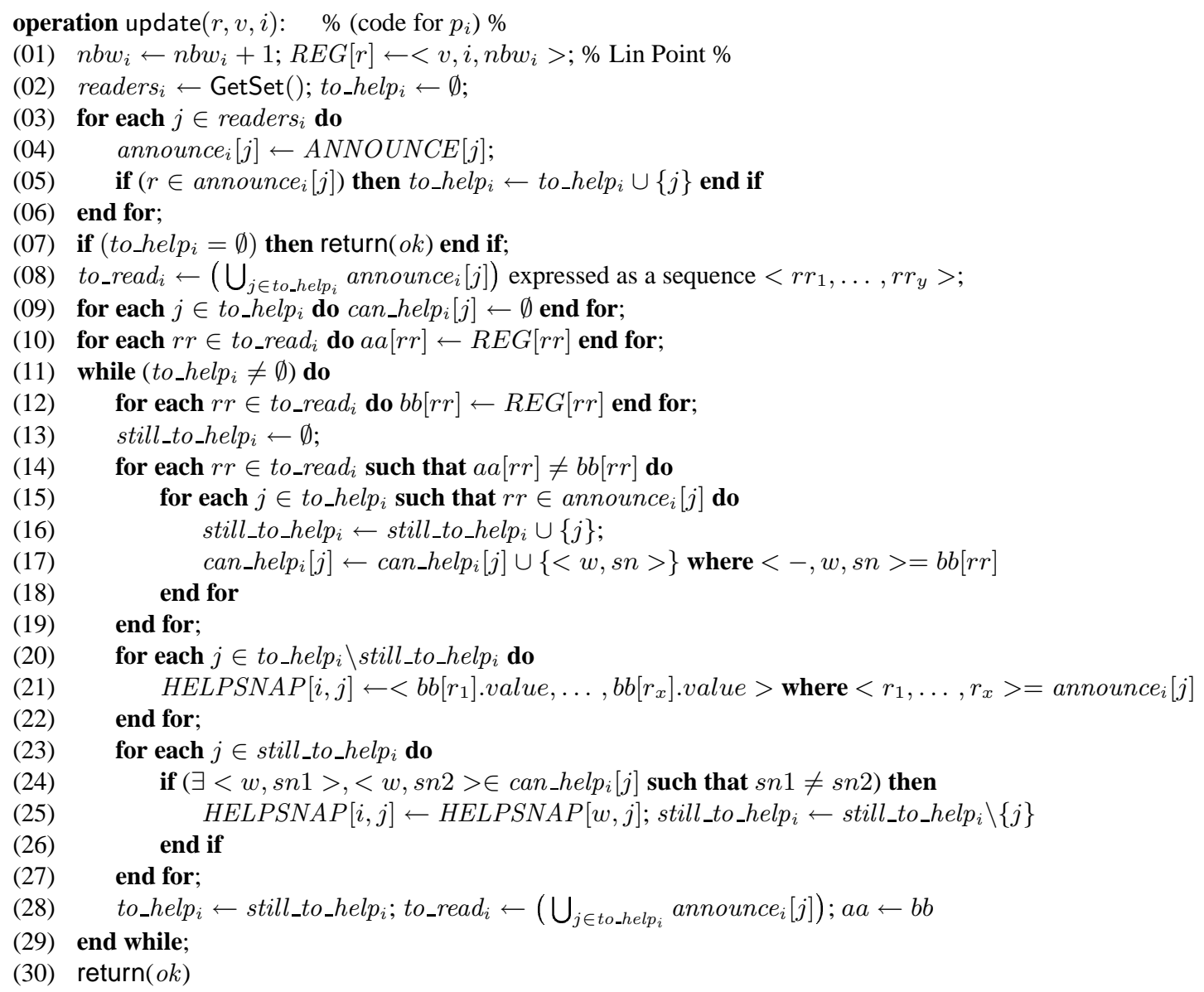

Figure 3: An algorithm for the update() operation

Are there processes to help? As in [8], a process $p_{i}$ first invokes GetSet () to learn the set of processes that have concurrently invoked a p_snapshot $(R)$ operation (line 02 ). It then computes the set to help $i$ of the conflicting processes, i.e., the ones such that $r \in R$. To that end, it uses the array ANNOUNCE (lines 03-06). If there is no conflicting process $\left(t_{-} h e l p_{i}=\emptyset\right), p_{i}$ does not have to help (help-locality property), and terminates accordingly (line 07 ).

If $t o \_h e l p_{i} \neq \emptyset, p_{i}$ has to possibly help the processes in to help $p_{i}$. To that end, it first computes the set to_read $_{i}$ of the components it has to read to help these processes (line 08). It also initializes a local array can_help $p_{i}$ to $\emptyset$ (line 09 ). The entry can_help $p_{i}[j]$ contains the processes $p_{w}$ that (to $p_{i}$ 's knowledge) could also help the conflicting process $p_{j}$. 
How a process helps individually another process Each process $p_{j}$ in $t o_{-} h e l p_{i}$ is helped individually by $p_{i}$. This is done in the loop (line 11-29), that terminates when the set to help $p_{i}$ becomes empty.

In each loop iteration, similarly to what is done in the p_snapshot () operation, $p_{i}$ first executes a double scan (whose values are kept in the local arrays $a a$ and $b b$ ) and does the following.

- Part 1: lines 13-19. For each component $r r$ such that $a a[r r] \neq b b[r r]$, let $b b[r r]=<-, w, s n>$, which means that (to $p_{i}$ 's knowledge) $p_{w}$ is the last process that wrote the component $\operatorname{rr}$ (lines 14 and 17). Moreover, this write occurred between the double scan. According to that observation, $p_{i}$ keeps track of the fact that such a process $p_{w}$ could help every $p_{j}$ such that $r r \in$ announce $_{i}[j]$. This is done by adding the pair $\left\langle w, s n>\right.$ to the set can_help $p_{i}[j]$ (lines 15-17). Additionally, $p_{i}$ adds $j$ to the set still_to_help $p_{i}$ (lines 13 and 16).

- Part 2: lines 20-22. Then, $p_{i}$ looks for the processes that can be helped directly. Those are the processes $p_{j}$ such that $j \in t_{0}{ }_{-} h e l p_{i} \backslash$ still_to_help $p_{i}$. The components $r r$ they want to read are such that $a a[r r]=b b[r r]$, which means that the pair $(a a, b b)$ constitutes a successful double scan. Accordingly, $p_{i}$ writes in HELPSNAP $[i, j]$ a snapshot value that $p_{j}$ can use if its partial snapshot operation is still pending. The value of this helping snapshot value is $\left\langle b b\left[r_{1}\right] . v a l u e, \ldots, b b\left[r_{x}\right] . v a l u e>\right.$ where $\left.<r_{1}, \ldots, r_{x}\right\rangle=$ announce $_{i}[j]$ (line 21$)$.

- Part 3: lines 23-27. For each process $p_{j}$ that it has not previously helped (line 23), $p_{i}$ looks if there is a process $p_{w}$ that can help $p_{j}$. The helping termination predicate (line 24) is the same as the one used in the p_snapshot () algorithm (line 13 in Figure 2): there are two writes issued by a process $p_{w}$ that appear in $c a n_{-} h e l p_{i}[j]$. If the predicate is true, the helping value provided to $p_{j}$ by $p_{w}$ is borrowed by $p_{i}$ to help $p_{j}$ (line 25).

- Part 4: line 28. Finally, $p_{i}$ updates to_help $p_{i}$ and to_read $d_{i}$ before entering again the loop. If to_help $p_{i}=$ $\emptyset$, the loop terminates.

\section{Proof of the algorithm}

\subsection{Preliminary definitions}

Definition 3 If a process terminates a partial snapshot operation at line 08 (Figure 2), or executes line 21 of an update operation (Figure 3), the last pair of readings of the array REG constitutes a successful double scan. (The first scan is the reading whose values are kept in the array aa[], while the second scan is the reading whose values are kept in the array $b b[]$.

Definition 4 Let psp be a partial snapshot operation issued by a process $p_{i}$.

- psp is 0-helped if it terminates with a successful double scan (Figure 2, line 08).

- psp is 1-helped if it terminates by returning HELPSNAP $[w 1, i]$ (Figure 2, line 15), and the values in HELPSNAP $[w 1, i]$ come from a successful double scan by $p_{w 1}$ (i.e., the values in HELPSNAP $[w 1, i]$ have been computed at line 21 of Figure 3 by an update issued by $\left.p_{w 1}\right)$.

- psp is 2-helped if it terminates by returning HELPSNAP[w1,i] (Figure 2, line 15), and the values in HELPSNAP $[w 1, i]$ come from HELPSNAP $[w 2, i]$ ( $p_{w 1}$ executed line 25 , Figure 3 ) which in turn come from a successful double scan by $p_{w 2}$ (i.e., the helping update by $p_{w 2}$ computed these values at line 21).

- For the next values of $h$, the $h$-helped notion is defined similarly.

The aim of the following definitions is to help prove that the values returned are "consistent", i.e., they are from the appropriate registers, were simultaneously present in the snapshot object and are recent ${ }^{5}$.

\footnotetext{
${ }^{5}$ Always returning the initial values would provides well-defined and mutually consistent values, but those would not be fresh values.
} 
Definition 5 The values $\left\langle v_{1}, \cdots, v_{y}\right\rangle$ returned by a p_snapshot $\left(\left\langle r_{1}, \ldots, r_{x}\right\rangle\right)$ operation are well defined if $x=y$ and for each $\ell, 1 \leq \ell \leq x$, the value $v_{\ell}$ has been read from $R E G\left[r_{\ell}\right]$.

Definition 6 The values returned by a p_snapshot $\left(\left\langle r_{1}, \ldots, r_{x}\right\rangle\right)$ operation are mutually consistent if there is a time at which they were simultaneously present in the snapshot object.

Definition 7 The values returned by a p_snapshot $\left(\left\langle r_{1}, \ldots, r_{x}\right\rangle\right)$ operation are fresh if, for each $\ell$, $1 \leq \ell \leq x$, the value $v_{\ell}$ returned for $r_{\ell}$ is not older than the last value written into $R E G\left[r_{\ell}\right]$ before the partial snapshot invocation ${ }^{6}$.

\subsection{The values returned are well-defined, mutually consistent and fresh}

Lemma 1 The values returned by a 0-helped p_snapshot() operation are well-defined, mutually consistent and fresh.

Proof Let p_snapshot $\left(\left\langle r_{1}, \ldots, r_{x}\right\rangle\right)$ be a 0-helped snapshot operation. As it terminates at line 08 , it follows that the value returned for each component $r \in\left\{r_{1}, \ldots, r_{x}\right\}$ has been obtained from $b b[r] . v a l u e$. The well-definition of these values follows directly from the observation that, for each $r \in\left\{r_{1}, \cdots, r_{x}\right\}$, the value currently in $b b[r]$ has been obtained at line 05 from the corresponding register $R E G[r]$.

For mutual consistency and freshness, let us notice that the termination of the p_snapshot() operation is due to a successful double scan. None of the values read has been modified between these two scans (otherwise the predicate of line 06 would be false). As these scans are sequential (the second one is started only after the first one has terminated) it immediately follows that, at any time between these two scans, the values that are returned were simultaneously present in the shared memory and, for each $v_{\ell}$, no write into $R E G\left[r_{\ell}\right]$ occurred between the write of $\left\langle v_{\ell},-,->\right.$ into $R E G\left[r_{\ell}\right]$ and its read whose value has been kept in $b b\left[r_{\ell}\right]$. It follows that the values returned are both mutually consistent and fresh, which proves the lemma.

Lemma 2 The values returned by a 1-helped p_snapshot() operation are well-defined, mutually consistent and fresh.

Proof Let psp $=$ p_snapshot $\left.\left(<r_{1}, \ldots, r_{x}\right\rangle\right)$ be the 1-helped snapshot operation and $p_{i}$ the process that invoked it. As that operation returns $\operatorname{HELPSNAP}[w 1, i]$ at line 15 , it follows that the predicate evaluated at line 13 is true: there is a process $p_{w 1}$ such that there are two distinct pairs $\left.\left.<w 1, s n 1\right\rangle,<w 1, s n 2\right\rangle \in$ can_help_me $e_{i}$. Without loss of generality let $s n 1<s n 2$. So, $w 1$ has been added twice to can help_me $e_{i}$ (line 11). The proof follows from the following sequence of observations. The time instants defined and used in the proof are depicted in Figure 4. Except when explicitly indicated, the line numbers refer to Figure 2.

1. As $w 1$ appears twice in $c a n \_h e l p \_m e_{i}$, it follows that the process $p_{w 1}$ has issued two distinct updates, say update $(r 1, v, w 1)$ and update $\left(r 2, v^{\prime}, w 1\right)$, that entailed two writes (to $R E G[r 1]$ and $R E G[r 2]$ ) such that $r 1, r 2 \in\left\{r_{1}, \ldots, r_{x}\right\}$ (let notice that it is possible that $r 1=r 2$ ).

2. It follows from the addition of $\left\langle w 1\right.$, sn $1>$ to $c a n \_h e l p \_m e_{i}$ (that is due to the component $r 1$ ), that there are two time instants $t_{1}$ and $t_{2}$, and two different values $a a 1$ and $b b 1$, such that:

- There is a process $p_{u}$ that has written $a a 1$ into $R E G[r 1]$, and that value has then been read by $p_{i}$ and stored in $a a[r 1]$ at time $t_{1}$ (line 03 or lines 05 and 17).

\footnotetext{
${ }^{6}$ Let us recall that, as each $R E G\left[r_{\ell}\right]$ is an atomic register, its read and write operations can be totally ordered in a consistent way. The word "last" is used with respect to this total order.

PI ${ }^{\circ} 1907$
} 


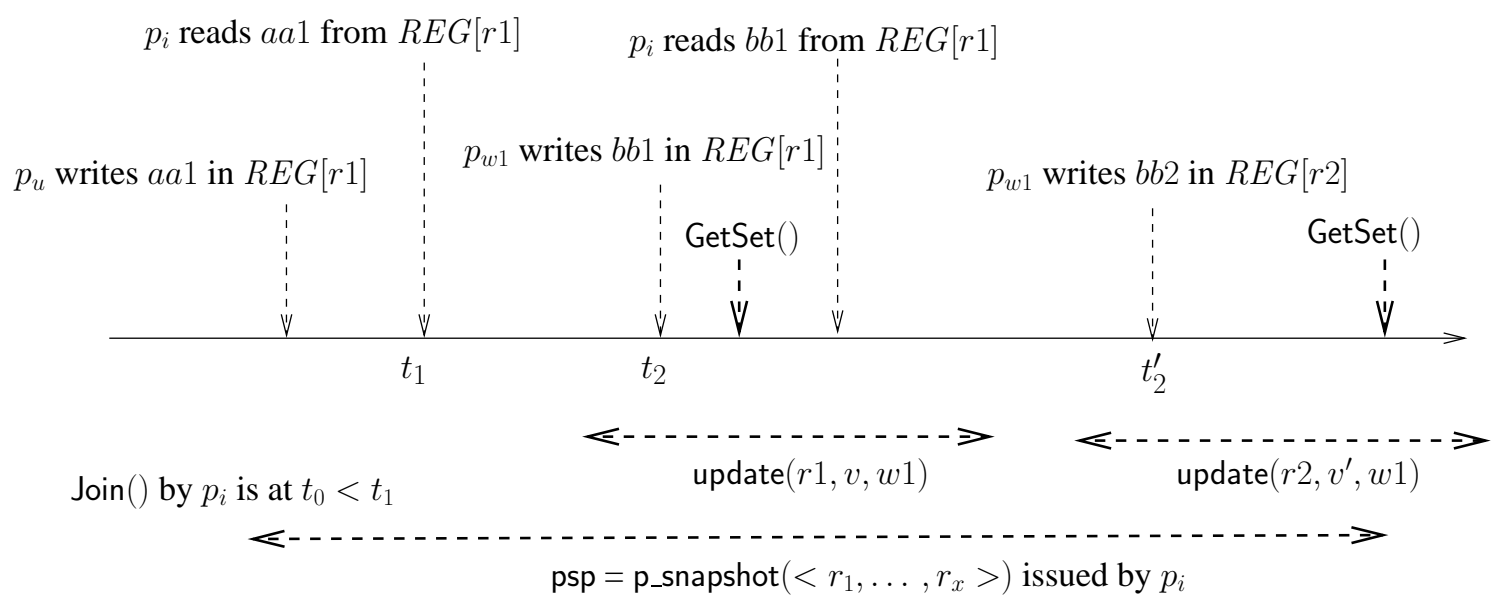

Figure 4: Order on operations on base objects

- $p_{w 1}$ has written $b b 1=<v, w 1, s n 1>$ into $R E G[r 1]$ at time $t_{2}$ (line 01 of Figure 3 ), and that value has later been read by $p_{i}$ and stored in $b b[r 1]$ (line 05 ).

- As $p_{i}$ reads from the atomic register $R E G[r 1]$ first $a a 1$ and then $b b 1 \neq a a 1$, it follows that $t_{1}<t_{2}$.

3. Similarly to the previous item, it follows from the addition of $\left\langle w 1, s n 2>\right.$ to can $_{\text {help }} \mathrm{me}_{i}$ (due to $r 2$ ) that there are two time instants $t_{1}^{\prime}$ and $t_{2}^{\prime}$, and two different values $a a 2$ and $b b 2$, such that:

- There is a process $p_{s}$ that has written $a a 2$ into $R E G[r 2]$, and that value has then been read by $p_{i}$ and stored in $a a[r 2]$ at time $t_{1}^{\prime}$ (line 03 or lines 05 and 17).

- $p_{w 1}$ has written $b b 2=<v^{\prime}, w 1, s n 2>$ into $R E G[r 2]$ at time $t_{2}^{\prime}$ such that $t_{1}^{\prime}<t_{2}^{\prime}$ (line 01 of Figure 3), and that value has later been read by $p_{i}$ and stored in $b b[r 2]$ (line 05 ).

4. As $p_{w 1}$ is sequential and $s n 1<s n 2$, it follows that $p_{w 1}$ has first executed update $(r 1, v, w 1)$ (that entailed the write of $R E G[r 1]$ ) before executing update $\left(r 2, v^{\prime}, w 1\right)$ (that entailed the write $R E G[r 2]$ ). It follows from that observation that $t_{2}<t_{2}^{\prime}$.

5. Let $t_{0}$ be the time at which $p_{i}$ started its Join() invocation (line 02). As $p_{i}$ executes Join() before reading entries of $R E G$, we have $t_{0}<t_{1}$, and consequently $t_{0}<t_{2}<t_{2}^{\prime}$.

6. When $p_{w 1}$ executed update $(r 1, v, w 1)$, it first wrote $R E G[r 1]$, and only then invoked GetSet () (lines 01-02 of Figure 3). As $t_{0}<t_{2}$, it follows from the specification of the underlying active set object that $i$ belongs to the set reader $s_{w 1}^{r 1}$ returned by the GetSet() invocation issued by update $(r 1, v, w 1)$.

7. The values returned by psp have been deposited in $\operatorname{HELPSNAP}[w 1, i]$ either by the first update update $(r 1, v, w 1)$ or the second update update $\left(r 2, v^{\prime}, w 1\right)$. Let up1 be this update. If they have been deposited by the second update, it follows from the lines 02-06 of Figure 3 that we necessarily have $i \in$ reader $s_{w 1}^{r 2}$.

The next item shows that, whatever the update that deposited in $\operatorname{HELPSNAP}[w 1, i]$ the values that are read by $p_{i}$, those are well defined, mutually consistent and fresh.

8. It follows from the following facts:

- up1 is on a component $r 1$ (or $r 2$ ) that $p_{i}$ wants to read (Item 1),

- The predicate $i \in$ reader $s_{w 1}$ is true when evaluated in up1,

- $p_{i}$ updates $A N N O U N C E[i]$ to $<r_{1}, \ldots, r_{x}>$ before invoking Join( $)$, 
- up1 invokes GetSet() (and obtains reader $s_{w 1}$ ) before reading the array ANNOUNCE, that up1 sets announce $w_{w 1}[i]$ to $<r_{1}, \ldots, r_{x}>$ and includes $i$ in to_help $p_{w 1}$ (lines 04-05 of Figure 3).

9. As psp is a 1-helped snapshot operation (assumption), the values read by psp from $\operatorname{HELPSNAP[w1,i]}$ have been deposited by up1 at line 21 (Figure 3). As they are the values of the components in announce $_{w 1}[i]$, they are well defined. Moreover, as they are from a successful double scan, they are mutually consistent. As the double scan started after the beginning of psp, they are fresh.

$\square_{\text {Lemma } 2}$

Lemma 3 For $h \geq 2$, the values returned by a h-helped partial snapshot operation are well-defined, mutually consistent and fresh.

Proof We state the proof for $h=2$. The proof of the cases $h \geq 3$ is obtained from a simple induction on $h$. Let psp $=$ p_snapshot $\left(\left\langle r_{1}, \ldots, r_{x}\right\rangle\right)$ be a 2 -helped partial snapshot operation that, invoked by a process $p_{i}$, returns the values in HELPSNAP $[w 1, i]$. These values have been written by $p_{w 1}$ in HELPSNAP $[w 1, i]$ at line 25 of an update operation (denoted upw1). Moreover, that write assigned to HELPSNAP $[w 1, i]$ the current value of HELPSNAP $[w 2, i]$, that has been computed at line 21 of an update (denoted upw2) issued by $p_{w 2}$. We have the following.

- The Items 1-8 stated in the proof of the Lemma 2 are still valid when considering psp and upw1. (This follows from the observation that only the Item 9 uses the fact that psp be a 1-helped partial snapshot operation.) It follows that $i \in$ readers $_{w 1}$ and announce $_{w 1}[i]=\left\langle r_{1}, \ldots, r_{x}\right\rangle$.

- Claim. upw2 is such that announce $_{w 2}[i]=<r_{1}, \ldots, r_{x}>$.

Proof of the claim. As $p_{w 1}$ executes HELPSNAP $[w 1, i] \leftarrow H E L P S N A P[w 2, i]$ (line 25, Figure 3), there are two components $r 1$ and $r 2$ such that (1) $r 1, r 2 \in$ announce $_{w 1}[i]=<r_{1}, \ldots, r_{x}>$. and (2) $p_{w 2}$ issued two updates involving $R E G[r 1]$ and $R E G[r 2]$, and (3) one of these updates is upw2.

As upw2 started after the beginning of upw1, that in turn started after the Join() invoked by psp, it follows that the set returned by the GetSet() invocation by upw2 includes $i$, and consequently, announce $_{w 2}[i]=<r_{1}, \ldots, r_{x}>$ (lines 02-06 and lines 20-22 of Figure 3, executed by upw2). End of the proof of the claim.

- If follows from the previous items and the fact that the values in $\operatorname{HELPSNAP}[w 2, i]$ are determined from a successful double scan at line 21 by upw2, that they are well-defined, mutually consistent and fresh.

$\square_{\text {Lemma } 3}$

\subsection{Uptodateness and help-locality}

Lemma 4 The update() and p_snapshot() algorithms satisfy the uptodateness and help-locality properties.

Proof The help-locality follows immediately from line 05 of the update algorithm. The uptodateness property follows from the fact that an update $(r, v,-)$ operation first writes $v$ into $R E G[r]$, and only then reads the value in $R E G[r]$ if it is needed for helping some processes.

$\square_{\text {Lemma } 4}$ 


\subsection{Wait-freedom}

The next lemma shows that the algorithms that construct a partial snapshot object are wait-free, i.e., terminate despite the crash of any number of processes [16].

Lemma 5 When executed by a correct process, every update() and p_snapshot () operation terminates.

Proof Let us recall that we assume a wait-free implementation of the underlying active set object. The proof is similar to the proof described in [1] designed for for a snapshot object whose components are single-writer/multi-reader registers.

Let us first consider a p_snapshot() invocation, issued by a correct process $p_{i}$. If, while $p_{i}$ executes line 06 , the test is true, the p_snapshot() operation terminates. So, let us assume that this test is never satisfied. We have to show that the predicate of line 13 eventually becomes true. As the predicate of line 06 is never satisfied, each time $p_{i}$ executes the loop body, there is a component $r$ such that $a a[r] \neq b b[r]$. The process $p_{k}$ that has modified $R E G[k]$ between the two readings by $p_{i}$ entails the addition of the pair $<k, s n k>$ to can_help_me $e_{i}$ (where $\langle k, s n k>$ is extracted from $b b[r]$ ). In the worst case, $n-1$ pairs (one associated with each process, but $p_{i}$ because it cannot execute an update operation while it executes a snapshot operation) can be added to $c a n \_h e l p \_m e_{i}$ while the predicate of line 13 remains false. But once $c a n \_h e l p \_m e_{i}$ contains one pair per process (but $p_{i}$ ), the next pair that is added is necessarily due to a process $p_{w}$ such that can_help_me $e_{i}$ already contains a pair $\langle w, s n 1\rangle$. Consequently, after line 11 has been executed due to that process $p_{w}$, a second pair $<w, s n 2>$ is added to can_help_me ${ }_{i}$. Then, the test of line 13 becomes satisfied, which proves the lemma.

Let us now consider an update() invocation, issued by a correct process $p_{i}$. If, when computed at line 05 , to_hel $p_{i}$ remains empty, $p_{i}$ terminates at line 07 . So, let us suppose that to $h e l p_{i} \neq \emptyset$, and $p_{i}$ executes the while loop (lines 11-29).

We have to show that the set $t o-h e l p_{i}$ eventually becomes empty. The processes that are not added to the set still_to_help $p_{i}$ (line 16) are suppressed from to_help $p_{i}$ (line 28). So, let us assume (by contradiction) that

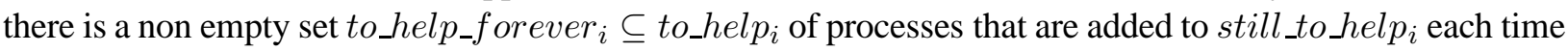
$p_{i}$ executes the body of the while loop (line 16). Let $p_{j}$ be one of these processes. As still_to help $p_{i}$ is reset to $\emptyset$ each time $p_{i}$ executes the loop body and $p_{j}$ remains forever in to_help_forever ${ }_{i}$, it follows that there is a pair $\langle w, s n\rangle$ that is added to can_help $_{i}[j]$ at each execution of the loop body. The reasoning is now the same as for proving the termination of the p_snapshot () algorithm. One new pair is added to can $_{h} h_{e l p}[j]$ each time $p_{i}$ executes the body of the loop and there are $n-1$ processes different from $p_{i}$. Consequently, after at most $n$ executions of the loop body, the new pair $\left\langle w^{\prime}, s n^{\prime}>\right.$ that is added to can_help ${ }_{i}[j]$ is such that $w=w^{\prime}$ and $s n \neq s n^{\prime}$. Then, the test of line 24 becomes satisfied and $p_{j}$ is suppressed from still_to_help $p_{i}$, contradicting the initial assumption, which completes the proof of the lemma. $\quad \square_{\text {Lemma } 5}$

\subsection{Linearizability}

As stated in the Introduction and Section 3.1, the consistency property of a snapshot object is linearizability. This means that all the update() and p_snapshot() operations issued by the processes during a run (except possibly the last operation issued by faulty processes), have to appear as if they were executed one after the other, each one being executed at some point of the time line between its start event and its end event.

Lemma 6 Every run of a partial snapshot object whose update() and p_snapshot() are implemented with the algorithms described in the Figures 2 and 3 , is linearizable. 
Proof The proof consists in associating with each operation op a single point of the time line (denoted $l p(\mathrm{op})$ and called its linearization point), such that

- $l p(o p)$ is between the beginning (start event) of op and its end (end event),

- No two operations have the same linearization point,

- The sequence of the operations defined by their linearization points is a sequential execution of the snapshot object.

So the proof consists in an appropriate definition of the linearization points. The linearization point of each operation (except possibly the last operation of faulty processes) is defined as follows:

- An update $(r,-,-)$ operation is linearized at the time of its embedded write of $R E G[r]$ (line 01$)$.

- The linearization of a snapshot operation psp $=p_{\text {_snapshot }}\left(\left\langle r_{1}, \cdots, r_{x}\right\rangle\right)$ depends on the line at which its return() statement is executed.

- Case 1: psp returns at line 08 due a successful double scan (psp is 0-helped). Its linearization point is any point of the time line between the first scan and the second scan of that successful double scan.

- Case 2: psp returns at line 15 , (psp is $h$-helped with $h \geq 1$ ). In that case, the values returned by psp have been computed by some update operation at line 21 or line 25 . Moreover, whatever the case, they have been computed by a successful double scan executed by some process $p_{z}$. When considering this successful double scan, $l p(\mathrm{psp})$ is placed between the end of the first scan and the beginning of the second one.

It follows from the previous definition of the linearization points that each operation is linearized between its beginning and its end, and no two operations are linearized at the same point ${ }^{7}$. Moreover, it follows from the Lemmas 1,2 and 3 concerning the well-definition, mutual consistency and freshness of the values returned by the partial snapshot operations, that the update and snapshot operations appear as if they had been executed according to the total order defined by their linearization points, which completes the proof of the lemma.

$\square_{\text {Lemma } 6}$

\subsection{The partial snapshot object is correct, wait-free and efficient}

Theorem 1 The algorithms described in Figure 2 and Figure 3 satisfy the termination and consistency properties (stated in Section 3.1) that defines a partial snapshot object. Moreover, they satisfy the uptodateness and help-locality properties.

Proof The proof is an immediate consequence of the lemmas 4, 5 and 6.

$\square$ Theorem 1

\section{Discussion}

\subsection{On the cost of the p_snapshot () and update () operations}

Evaluating the "real" cost of the operations is difficult. This is mainly due to two reasons. The first one is the difficulty to figure out realistic patterns that address both (1) the concurrency among the p_snapshot $(R)$ and

\footnotetext{
${ }^{7}$ If two operations are linearized at the same point, they can be tie-broken according to the id of the processes that issued these operations.
}

$\operatorname{PI} n^{\circ} 1907$ 
update $(r,-,-)$ operations, and, for each operation, (2) the content of its set $R$ or component $r$. The second one is the difficulty to find a "good" measure. Evaluating only the number of shared memory accesses is a poor measure, as it takes into account neither the size of the values that are atomically read or written (that defines an "atomicity grain" of the corresponding read and write operations), nor the restrictions on asynchrony imposed by that "atomicity grain" .

Such a "real cost" analysis is beyond the scope of this paper (whose main concern is the investigation of new properties and new techniques for implementing snapshot objects). The following analysis, that only counts the number of shared memory accesses, is consequently limited and should not be considered as an ultimate cost criterion. It has to be enriched with other measures to become meaningful.

Cost of the p_snapshot() operation Let us first look at the number of accesses to the array $R E G$. It follows from the proof of Lemma 5 that, in the worst case, a p_snapshot () operation executes $n$ times the body of its while loop (lines 04-18). Consequently, it reads (at lines 03 and 05, Figure 2) $n+1$ times the $x$ components $r_{1}, \cdots, r_{x}$ it is interested in. Hence, as far the array $R E G$ is concerned, there are at most $(n+1) x$ shared memory accesses. This is particularly interesting when $n<m$, i.e., when the number of processes is much smaller than the number of components (a case that occurs when a lot of critical variables are kept in the same snapshot object).

In the best case, a p_snapshot() operation reads only twice the $x$ entries of the array $R E G$ in which it is interested. In these cases there are only $2 x$ accesses of the array $R E G$. Interestingly, this can appear in two distinct scenarios.

- The first scenario is when the p_snapshot () terminates due to a successful double scan that occurs during the first execution of the loop.

- The second scenario is when, during the first execution of the loop, the predicate of line 13 is true (while the one of line 06 is false). This occurs when a process $p_{w}$ has updated two different components $r 1$ and $r 2$ such that $r 1, r 2 \in\left\{r_{1}, \cdots, r_{x}\right\}$, and these updates occurred between the scans of $R E G$ in $a a$ and $b b$, respectively.

In addition to the accesses to the array $R E G$, a p_snapshot() operation accesses once the array $A N N O U N C E$, and at most once the array HELPSNAP. It also invokes once Join() and Leave().

Cost of the update() operation An update() operation writes a single register $R E G[r]$ and invokes once GetSet(). Then its number of shared memory accesses depends on the value readers ${ }_{i}$ returned by GetSet() to the invoking process $p_{i}$.

If the set readers $s_{i}$ is empty, there are no concurrent p_snapshot() operations, and update() terminates. Otherwise, let $\alpha=\mid$ readers $_{i} \mid$. Then, $p_{i}$ accesses $\alpha$ times the shared memory to read appropriate entries of the array $A N N O U N C E$. Let $\beta=\mid$ to_hel $_{i} \mid$. If $\beta=0$, there is no more shared memory accesses. If $\beta \neq 0$, the update() operation accesses $\beta$ times the vector $\operatorname{HELPSNAP}[i,-]$. The number of accesses to the array $R E G$ depends on the size of $t_{\_} \_$help $p_{i}$. In the worst case there are $n\left(\mid\right.$ to_read $\left._{i} \mid\right)$ accesses to the array $R E G$.

\subsection{Balancing the load: an active set per component}

The p_snapshot () and update () algorithms presented in Figure 2 and Figure 3, respectively, use a single active set object. A direct consequence is the fact that, when a process $p_{i}$ that executes the update $(r,-,-)$

\footnotetext{
${ }^{8}$ Using large atomic registers that contain large values can be more costly than using several atomic registers of smaller size (e.g., $\operatorname{HELPSNAP}[i, j]$ registers).
} 
algorithm invokes GetSet () , it obtains a set readers ${ }_{i}$ made up of the ids of all the processes that are concurrently executing a p_snapshot $\left(R_{\ell}\right)$ operation, independently of the fact that $r$ belongs or not to the corresponding sets $R_{\ell}$. The process $p_{i}$ has then to read all the registers $A N N O U N C E[j]$ for $j \in \operatorname{GetSet}()$ in order to learn which are the ones such that $r \in R_{\ell}$ (lines 03-06 in Figure 3). This places on each update operation a load (in number of shared memory accesses) that is due to the set of components read by the partial snapshot operations.

There is a simple way to balance this load by using an active set per component of the partial snapshot object. Let $A S[1 . . m]$ be the corresponding array of active set objects. We have the following modifications.

- The lines 01-03 of Figure 2 are replaced by the following lines:

ANNOUNCE $[i] \leftarrow<r_{1}, \cdots, r_{x}>$; can_help_me $e_{i} \leftarrow \emptyset$;

for each $r \in\left\{r_{1}, \cdots, r_{x}\right\}$ do $A S[r]$.Join(); $a a[r] \leftarrow R E G[r]$ end for.

- The lines 07 and 14 of Figure 2 are replaced by the following statement:

for each $r \in\left\{r_{1}, \cdots, r_{x}\right\}$ do $A S[r]$.Leave() end for.

- The lines 02-06 of Figure 3 are replaced by the following ones:

to_help $p_{i} \leftarrow A S[r]$.GetSet();

for each $j \in$ to_help $_{i}$ do announce $_{i}[j] \leftarrow A N N O U N C E[j]$ end for.

\subsection{Using $\mathrm{LL} / \mathrm{SC}$ registers instead of read/write atomic registers}

An array of LL/SC registers This section shows that using LL/SC registers instead of the atomic read/write registers as underlying base registers reduces the number of base registers from $O\left(n^{2}\right)$ to $O(n)$. More precisely, the array ANNOUNCE[1..n] and the matrix HELPSNAP $[1 . . n, 1 . . n]$ both made up of atomic read/write registers can be replaced by a single array $A N N H E L P[1 . . n]$ such that each of its registers is accessed by the pair of LL/SC operations.

The LL/SC pair of operations An LL/SC register is an atomic register that provides the processes with two operations denoted LL() (Linked Load) and SC() (Store Conditional). Considering an LL/SC register $X, X$.LL() returns the current value of $X$. A conditional store $X . \mathrm{SC}()$ issued by a process $p_{i}$ returns true (the write succeeded) or false (the write failed). Its success depends on the fact that, since the previous $X$.LL() issued by $p_{i}$, other processes have or have not updated $X$. It succeeds if and only if, since its last reading (whose value has been obtained by $X . \operatorname{LL}()), X$ has not been written by another process $p_{j}$ (whatever the value written by $p_{j}$, that value being possibly the same as the current value of $X$ ). If $X$.SC() is successful, $p_{i}$ knows that $X$ has not been updated since its last reading of $X$.

The input/output behavior of $X . \operatorname{LL}()$ and $X . \mathrm{SC}()$ can be precisely described by the following statements executed atomically (this description is from [24]). An array valid $_{X}[1 . . n]$ (initialized to $\left[\right.$ false $_{1}, \ldots$, false]) is associated with each LL/SC object $X ;$ valid $_{X}[i]$ is a flag set up by $p_{i}$ when it issued $X$. LL() and set down by any process $p_{j}$ when its write succeeds.

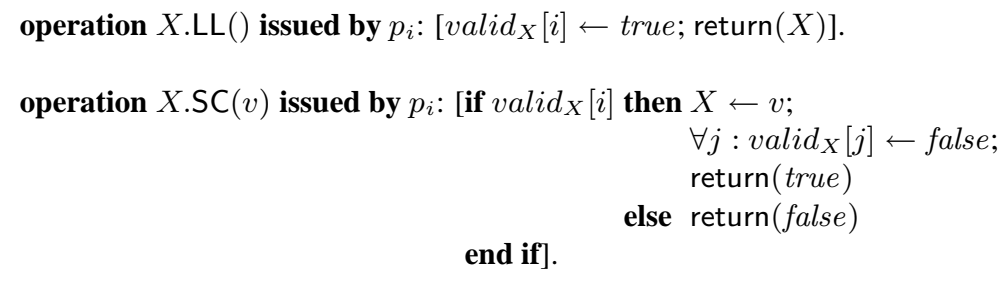

PI n ${ }^{\circ} 1907$ 
Weak variants of LL/SC registers are proposed in some architectures such as Alpha AXP (under the name Idl_l/stl_c), IBM PowerPC (under the name Iwarx/stwcx), or ARM (under the name Idrex/strex) [17]. In these architectures, the entities using these base operations are the processors (and not the processes).

The computational power of the pair LL/SC operations in presence of process crash failures is the same as the one of the Compare\&Swap operation, namely it has a consensus number equal to $+\infty$ [16].

The array $A N N H E L P[1 . . n] \quad$ The entry $A N N H E L P[i]$ is used both by $p_{i}$ and by any other process $p_{j} \neq p_{i}$ to pass information from one to the other (in both directions). It can contain three types of values, as described below.

- When it invokes p_snapshot $(R)$, the process $p_{i}$ sets $A N N H E L P[i]$ to $<r e q, R>$ to announce that it wants to read atomically the components of $R$.

- When it returns from p_snapshot $(R)$ without being helped (successful double scan), the process $p_{i}$ sets $A N N H E L P[i]$ to $\perp$ to prevent future help from any other process.

- When it helps $p_{i}$, a process $p_{j}$ writes into $A N N H E L P[i]$ the values corresponding to the components $R$ that $p_{i}$ wants to read.

So, $\left\langle\right.$ req, $R>, \perp$ and $\left\langle v_{1}, \ldots, v_{x}\right\rangle$ are the three types of values that $A N N H E L P[i]$ can contain. Its initial value is $\perp$.

The LL/SC-based update() operation The LL/SC-based update() operation is described in Figure 5. It is exactly the same as Figure 3 except for 4 lines that are modified (their line number is postfixed with "M"). More precisely, we have the following. Let $p_{i}$ be the process that executes update $(r, v, i)$.

- Lines 04.M and 05.M. The process $p_{i}$ now invokes $A N N H E L P[j] . \operatorname{LL}()$ to learn the announce of each concurrent reader $p_{j}$. If $A N N H E L P[j] . \operatorname{LL}() \neq<r e q,->$, then $p_{i}$ does not have to help $p_{j}$. This is because $p_{j}$ has already been helped by another process $p_{k}$ (in that case $A N N H E L P[j]$ contains a list of values) written by $p_{k}$ at line 21.M), or $p_{j}$ has terminated its p_snapshot $(R)$ operation without being helped by another process (in that case, as we will see in the algorithm of the p_snapshot () operation, $A N N H E L P[j]=\perp)$.

- Line 21.M. As just indicated, this line is executed when $p_{j}$ helps $p_{i}$. To that end, $p_{i}$ executes ANNHELP $[j] . \mathrm{SC}\left(<b b\left[r_{1}\right]\right.$.value, $\ldots, b b\left[r_{x}\right]$.value $\left.>\right)$ to store into ANNHELP $[j]$ the help it gives to $p_{j}$. Due to the semantics of the pair LL/SC of each register $A N N H E L P[j]$, the invocation $A N N H E L P[j] . \mathrm{SC}()$ issued by $p_{i}$ at line 21.M is successful only if (1) no other process has helped $p_{j}$ since the last invocation $A N N H E L P[j] . \mathrm{LL}()$ issued by $p_{i}$, and (2) $p_{j}$ has not yet terminated its partial snapshot. This ensures that $p_{i}$ does not overwrite a new announce by $p_{j}$.

- Line 25.M. Differently from the algorithm based on atomic read/write registers, where the help of $p_{i}$ to $p_{j}$ is individual and is consequently written in the entry $(i, j)$ of the matrix HELPSNAP (line 25 in Figure 3), the help of $p_{i}$ to $p_{j}$ is now written directly in $A N N H E L P[j]$ at line 21.M. Consequently, if the test of line 24 is satisfied, another process $p_{w}$ has already helped $p_{j}$, and $p_{i}$ does not have to relay this help anymore. Hence, line 25.M is line 25 without the relay of the help to $p_{j}$.

The LL/SC-based p_snapshot () operation The LL/SC-based p_snapshot $(R)$ operation is described in Figure 5. It is the exactly same as Figure 2 except for the two lines that are modified (their line number is postfixed with "M") and a new line that is added (its line number is postfixed by "A"). More precisely, we have the following. Let $p_{i}$ be the process that executes p_snapshot $(R)$. In order to make the presentation easier to follow, the line 15.M is first explained, then the line 07.A, and finally the line 01.M. 


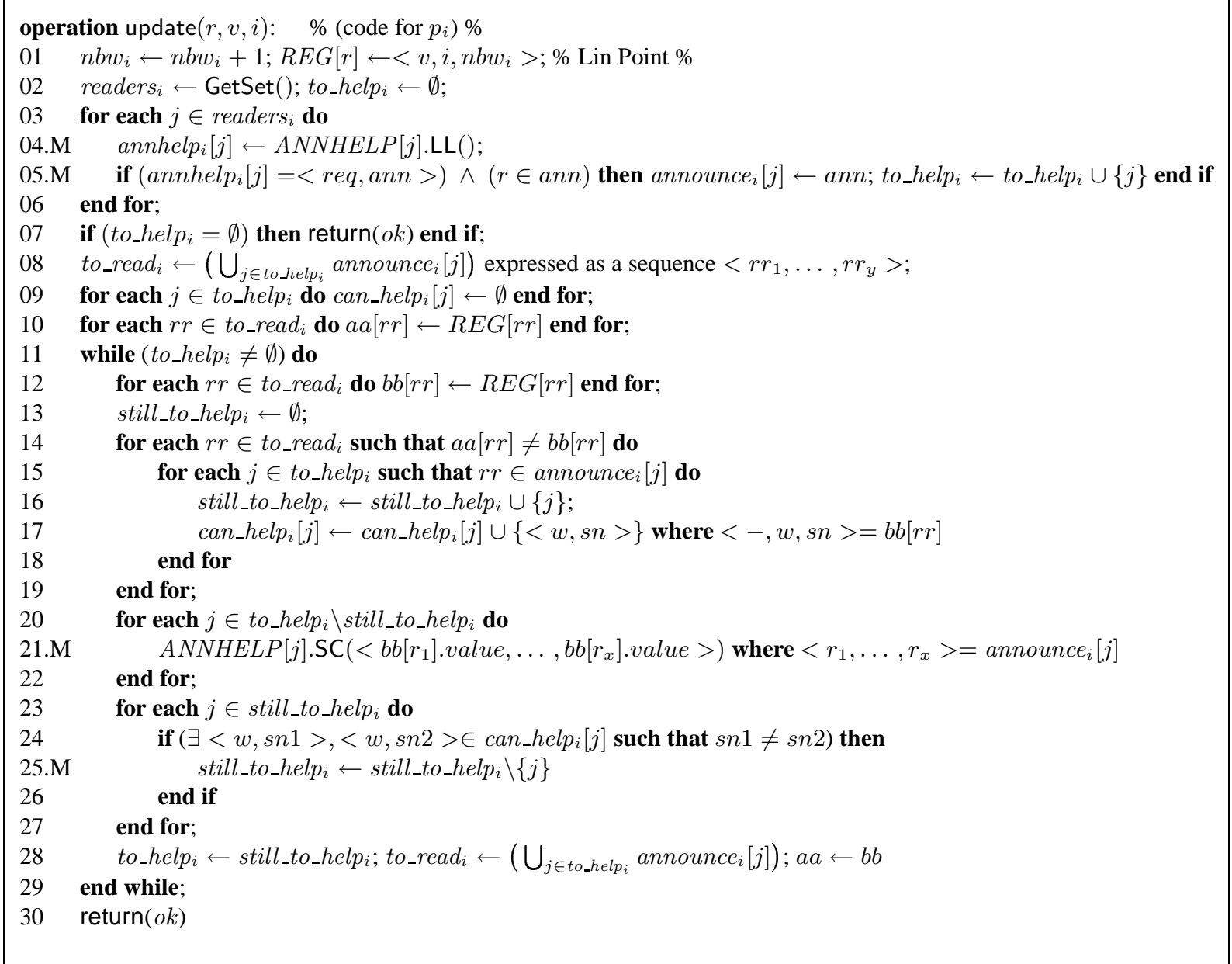

Figure 5: A LL/SC-based update() operation

- Line 15.M. If $p_{i}$ is helped by another process $p_{w}$, that process has deposited the helping values for $R$ in ANNHELP $[i]$. Consequently, $p_{i}$ returns these values for the components it has specified in $R$.

- Line 07.A. If $p_{i}$ terminates its partial snapshot operation without being helped by another process (successful double scan), it strives to write $\perp$ into $A N N H E L P[i]$. Let us observe that this write is successful if no process has deposited a helping value in $A N N H E L P[i]$.

In that way, when $p_{i}$ terminates its partial snapshot operation (being helped at line 15.M, or without help at line 08), we necessarily have ANNHELP $[i] \neq<r e q,->$ whatever the failure and asynchrony pattern. This means that, between the end of the current $p_{-}$snapshot () invocation issued by $p_{i}$ and the beginning of its next p_snapshot() invocation, no process $p_{i}$ can help it (as for $p_{j}$ to help $p_{i}$, $p_{j}$ has to find $A N N H E L P[i] \cdot \operatorname{LL}()=<r e q,->$, as described at line 05.M in Figure 5).

- Line 01.M. When $p_{i}$ calls p_snapshot $(R)$, it first invokes $A N N H E L P[i] . S C(<r e q, R>)$ to announce the components it wants to read. Let us observe that, due to the observation done in the previous item, the sequence LL/SC issued at line 01.M ensures that this conditional store is always successful. 


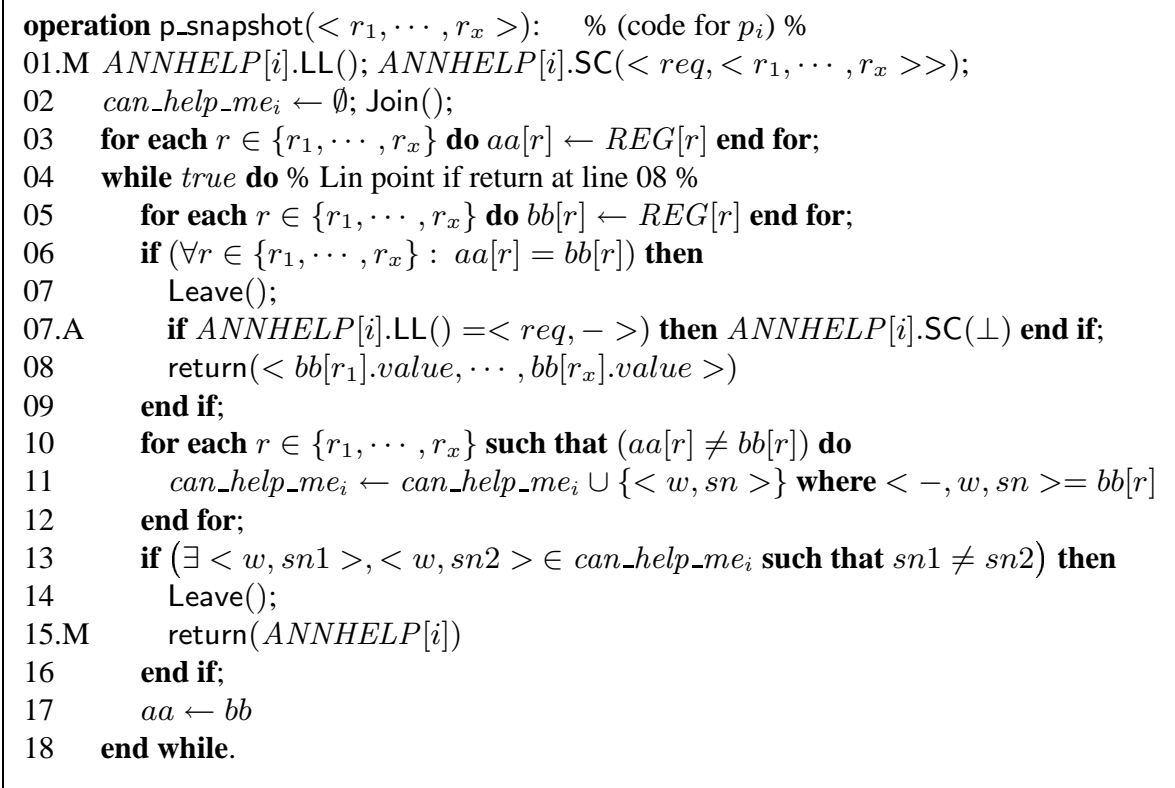

Figure 6: A LL/SC-based p_snapshot() operation

\section{Conclusion}

The concept of shared memory snapshot object has first been proposed in [1]. The notion of partial snapshot object has then been introduced in [8]. The present paper has first proposed two efficiency properties related to the implementation of partial snapshot objects. It has then addressed the design of a partial snapshot object whose implementation meets these properties. To attain this goal, the proposed implementation takes into account the current concurrency pattern and strives to be as efficient as possible. Its main features are the following 9 .

1. The proposed algorithm is the first that (to our knowledge) relies on the "write first, help later and help individually" strategy.

2. An update operation helps a snapshot operation only if needed, and no more. More explicitly, an update helps only the concurrent snapshots that read the component it writes. This new property, called help-locality states "no help when no conflict".

3. Differently from the update algorithms proposed so far, an update provides the processes with more asynchrony: the write of a new value and the writes of helping snapshot values are separated writes.

4. The update operation satisfies the following uptodateness property: the helping values (if any) supplied by an update $(r, v,-)$ operation includes, for the component $r$, the value $v$ (written by that update) or a more recent value.

5. The number of underlying base atomic registers can be reduced from $O\left(n^{2}\right)$ to $O(n)$ when these registers can be accessed by the LL/SC pair of operations instead of the weaker read/write pair of base operations.

\footnotetext{
${ }^{9}$ These features are also the main differences with the partial snapshot algorithm (based on read/write atomic registers) presented in [8], and the algorithms (based on read/write atomic registers) that implement a classical snapshot object (e.g., [1]).
} 


\section{References}

[1] Afek Y., Attiya H., Dolev D., Gafni E., Merritt M. and Shavit N., Atomic Snapshots of Shared Memory. Journal of the ACM, 40(4):873-890, 1993.

[2] Afek Y., Stupp G., Touitou D., Long-lived Adaptive Collect with Applications. Proc. 40th IEEE Symposium on Foundations of Computer Science Computing (FOCS'99), IEEE Computer Press, pp. 262-272, 1999.

[3] Aguilera M.K., A Pleasant Stroll Through the Land of Infintely Many Creatures. ACM SIGACT-NEWS, 35(2):36$59,2004$.

[4] Anderson J., Multi-writer Composite Registers. Distributed Computing, 7(4):175-195, 1994.

[5] Attiya H., Needed: Foundations for Transactional Memory. ACM SIGACT News, 39(1):59-61, 2008.

[6] Attiya H. and Fouren A., Algorithms Adapting to Contention Point. Journal of the ACM, 50(4):444-468, 2003.

[7] Attiya H., Fouren A. and Gafni E., An Adaptive Collect Algorithm with Applications. Distributed Computing, 15:87-96, 2002.

[8] Attiya H., Guerraoui R. and Ruppert E., Partial Snapshot Objects. Proc. 20th ACM Symposium on Parallel Architectures and Algorithms (SPAA'08), ACM Press, pp. 336-343, 2008.

[9] Attiya H. and Rachman O., Atomic Snapshot in $O(n \log n)$ Operations. SIAM Journal of Computing, 27(2):319340, 1998.

[10] Attiya H. and Welch J., Distributed Computing: Fundamentals, Simulations and Advanced Topics, (2d Edition), Wiley-Interscience, 414 pages, 2004.

[11] Chandy K.M. and Lamport L., Distributed Snapshots: Determining Global States of Distributed Systems. ACM Transactions on Computer Systems, 3(1):63-75, 1985.

[12] Ellen F., How Hard Is It to Take a Snapshot? Proc. 31th Conference on Current Trends in Theory and Practice of Computer Science (SOFTSEM'05), Springer-Verlag \#3381, pp. 28-37, 2005.

[13] Felber P., Fetzer C., Guerraoui R. and Harris T., Transactions Are Back-But Are They the Same? ACM SIGACT News, 39(1): 47-58, 2008.

[14] Guerraoui R. and Kapałka M., On the Correctness of Transactional Memory. Proc. 13th ACM SIGPLAN Symposium on Principles and Practice of Parallel Programming (PPoPP'08), ACM Press, pp. 175-184, 2008.

[15] Guerraoui R. and Raynal M., From Unreliable Objects to Reliable Objects: the Case of Atomic Registers and Consensus. 9th Int'l Conference on Parallel Computing Technologies (PaCT'07), Springer Verlag LNCS \#4671, pp. 47-61, 2007.

[16] Herlihy M.P., Wait-Free Synchronization. ACM Transactions on Programming Languages and Systems, 13(1):124-149, 1991.

[17] Herlihy M.P. and Shavit N., The Art of Mutiprocessor Programming. Morgan Kaufmann Pub., San Francisco (CA), 508 pages, 2008.

[18] Herlihy M.P. and Luchangco V., Distributed Computing and the Multicore Revolution. ACM SIGACT News, 39(1): 62-72, 2008.

[19] Herlihy M.P. and Wing J.M., Linearizability: a Correctness Condition for Concurrent Objects. ACM Transactions on Programming Languages and Systems, 12(3):463-492, 1990. 
[20] Imbs D. and Raynal M., A Lock-based STM Protocol that Satisfies Opacity and Progressiveness. Proc. 12th Int'l Conference On Principles Of Distributed Systems (OPODIS'08), Springer-Verlag LNCS \#5401, pp. 226$245,2008$.

[21] Inoue I., Chen W., Masuzawa T. and Tokura N., Linear Time Snapshots Using Multi-writer Multi-reader Registers. Proc. 8th Int'l Workshop on Distributed Algorithms (WDAG'94), Springer-Verlag \#857, pp. 130-140, 1994.

[22] Jayanti P., An Optimal Multiwriter Snapshot Algorithm. Proc. 37th ACM Symposium on Theory of Computing (STOCS'05), ACM Press, pp. 723-732, 2005.

[23] Lynch N.A., Distributed Algorithms. Morgan Kaufmann Pub., San Francisco (CA), 872 pages, 1996.

[24] Moir M., Practical Implementation of Non-Blocking Synchronization Primitives. Proc. 16th ACM Symposium on Principles of Distributed Computing (PODC'97), ACM Press, pp. 219-228, 1997.

[25] Shavit N. and Touitou D., Software Transactional Memory. Distributed Computing, 10(2):99-116, 1997.

[26] Taubenfeld G., Synchronization Algorithms and Concurrent Programming. Pearson Prentice-Hall, ISBN 0-13197259-6, 423 pages, 2006.

\section{A Full snapshot based on the "write first, help later" strategy}

The snapshot() and update() algorithms described in Figure 7 consider the case where each snapshot operation reads all the components. Moreover, an update systematically computes a helping snapshot value (i.e., whatever the concurrency pattern). These algorithms provide a basic implementation of a multi-writer/multireader snapshot object. They can be seen as a simplified (and not efficient) version of the algorithms presented in the body of the paper (Figures 2 and 3), that is the "write first, help later" counterpart of the classical snapshot algorithms presented in [1].

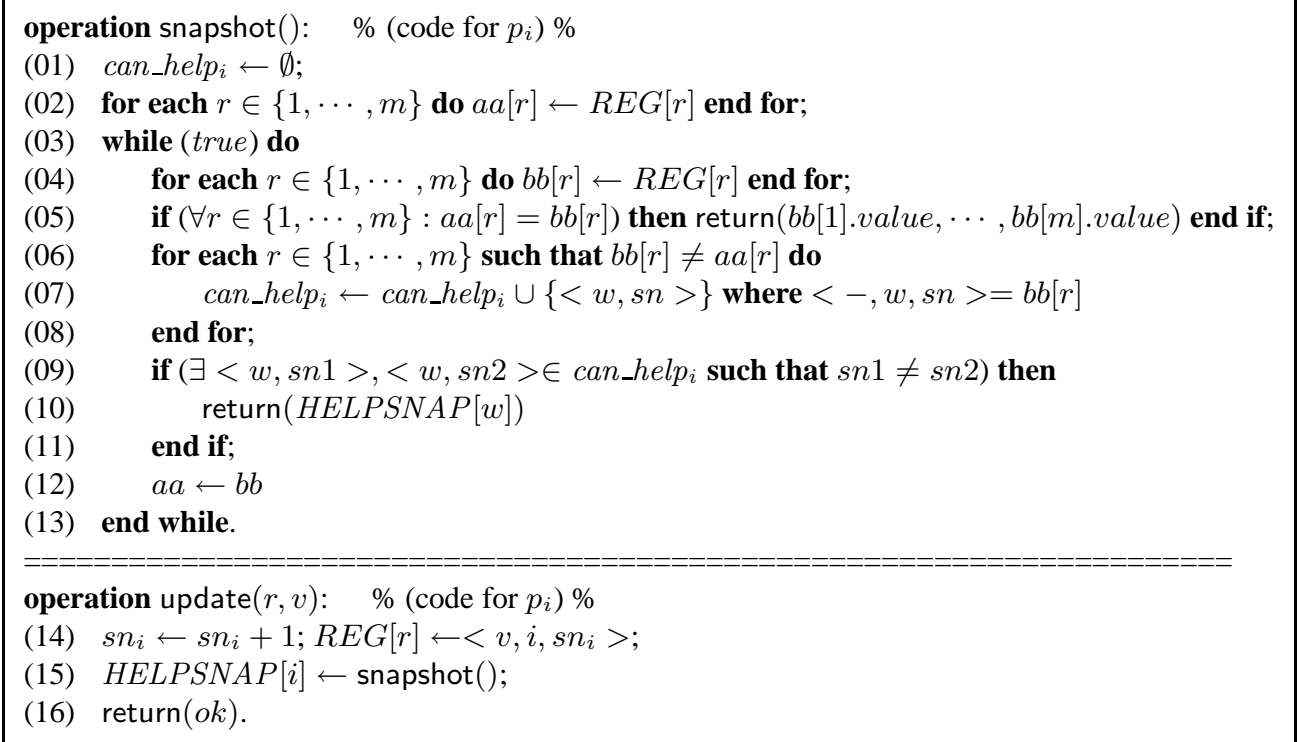

Figure 7: Algorithms for update and full snapshot 